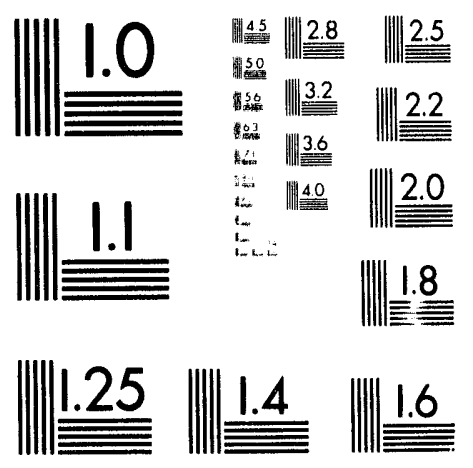



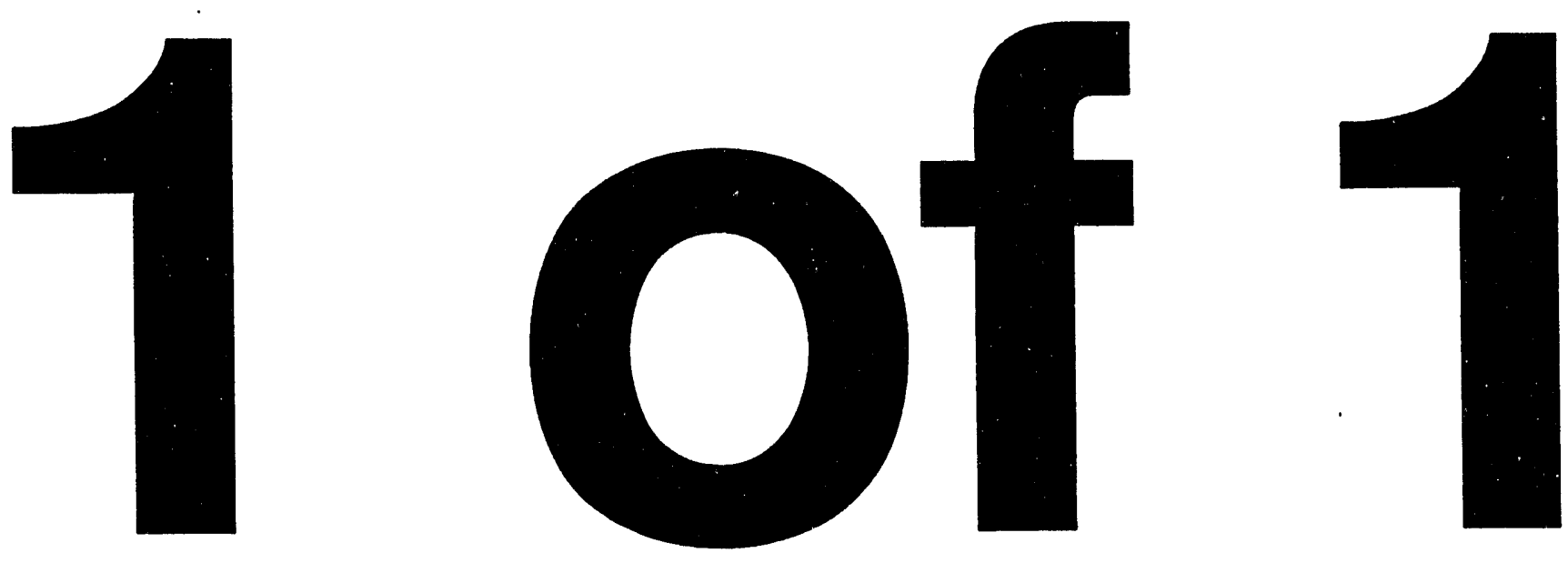
LBL-34729

CBP Note 042

UC-414

\title{
On a Theory of Two-Beam Mechanisms of Charged Particle Acceleration in Electrodynamic Structures*
}

\author{
A.O. Ostrovsky \\ Kharkov Institute of Physics and Technology, Kharkov 310108 Ukraine \\ (Supported by Accelerator and Fusion Research Division, Lawrence Berkeley Laboratory, \\ University of California, Berkeiey, California 94720)
}

September 1993

- Work supported in part by the Director, Office of Energy Research, Office of High Energy and Nuclear Physics, Division of High Energy Physics, of the U.S. Department of Energy under Contract No. DE-AC03$76 \mathrm{SF} 00098$ 
On a Theory of Two-Beam Mechanisms of Charged Particle Acceleration in Electrody.amic Structures

A.O. Ostrovsky

Kharkov Institute of Physics and Technclogy, Kharkov 310108 Ukraine

\section{Introduction}

This work is devoted to the theoretioal studies of two-beam meohanisms of charged partiole aoseleration in eleotrodynamio structures. The first section continues the outline of results of theoretical studies commenced in the intermediate report and considers the two-beam scheme of acceleration in the plasma waveguide. Aocording to this scheme the strong current relativistio eleotron beam (REB) (driving beam) exoites the intensive plasma waves acoelerating the electrons of the second beam (driven beam). The driving beam is assumed to be density-modulated. The preliminary modulation of the driving RFB is shown to enhance substantially the acceleration efficiency of relativistic electrons of the driven beam.

The second section deals with the two-beam aoceleration in the vacuum corrugated waveguide. According to this soheme the excitation of electromagnetic waves and aoceleration of driven beam electrons by them is accomplished under different Cherenkov resonances between the particles of beams and the corrugated waveguide field (tworesonance acceleration scheme) [1-3]. The electromagnetic field in the periodic structure is known to be the superposition of spatial harmonics. With the small depth of the periodic nonuniformity the amplitudes of these harmonics decrease fast with their number increasing [4]. Therefore, if the driving beam is in the Cherenkov resonanoe with the first spatial harmonic and the driven beam is in resonance with the zero space harmonic then the force accelerating the driven beam would be considerably bigger than the force deoelerating the driving beam electrons. One may expect in this case that the energy of accelerated particles at the system exit would exceed their initial value considerably. Ref.[2] has suggested to apply the waveguide with not very deep ormugated lateral surface as the accelerating structure. The main advantage of corrugated waveguides against vacuum periodic struotures of other types consists in their high electric strength as well as in the option to provide the favourable condition for the efficient interaction of strong current REB with the RF field of the structure. Cormugated waveguides have found a broad application in powerful relativistic 
microwave backward oscillators (BWO) [5,6]. Application of corrugated waveguides also permits to advance to the short wavelength range of accelerating fields $(\lambda \leqslant 1 \mathrm{~cm})$ and thereby to enhance their limiting values. This basioally must provide high acceleration rate for charged particles Tr100 $\mathrm{Mev} / \mathrm{m}$.

1. Aoceleration of oharged partioles by the modulated $\mathrm{R} F \mathrm{~B}$ in the plasma waveguide

In the intermediate report the two-beam scheme of accelerating relativistic eleotrons in the plasma waveguide has been considered. The most efficient acoeleration of eleotrons has been shown to ocour at a distance corresponding, approximately, to the distance to the first maximum of the field amplitude. At the same time the delayed exponential growth of the field amplitude in the starting region of the interaction space has been shown to limit essentially the acceleration rate of electrons. In this region comprising around $30 \%$ of the total length of the accelerating gap the field amplitude is small (linear stage of the instability evolution) and therefore the process of energy increase is practically absent. To decrease the region of linear stage of the instability evolution (region to the first maximum of the field amplitude) and thereby to increase the acceleration rate of electrons it is of interest to consider the case when the driving REB is preliminarily density-modulated. In this oase already formed bunches of electrons enter the interaction space. If the REB modulation frequency coincides with the frequency of the amplified wave the process of gathering electrons in bunohes oocurring at the linear stage of instability for the non-modulated beam would be absent. As a result, the distance at which the amplitude of the plasma wave achieves the first maximum must be considerably less than one for the non-modulated REB.

This seotion considers the acceleration of charged particles by the modulated REB in the plasma waveguide. At the waveguide entrance the density of the driving REB varies according to the harmonic law:

$$
n_{h}(z=0)=N_{b}\left(1+g \cos \omega_{m} t\right) \frac{\delta\left(r-r_{1}\right)}{2 \pi r_{1}},
$$

where $N_{b}$ is the beam particle density per unit length; $g$ is the 
modulation depth, $\omega_{m}$ is the modulation irequency. All the rest of variables and parameters in this section coincide with those given in the intermediate report. In what follows we assume the modulation frequency $\omega_{\mathrm{m}}$ to be equal to the frequency $\omega$ of the amplified plasma wave and the beam to be in exact Cherenkov resonance with this wave $(\sigma=0)$.

The set of equations describing the excitation of plasma waves and acoeleration of the driven RFB's particles corresponds completely to the set of equations (20)-(24) at $\sigma=0$ given in the intermediate report. The only difference from the non-modulated REBB consists in that $\rho$ value entering Eqs. (20), (21), (23) assumes now $2 \pi$

the form: $\rho=\int_{0} \Phi\left(\theta_{0}\right) \exp (1 \theta) d \theta_{0}$, where $\Phi\left(\theta_{0}\right)=1+g \cos \theta_{0}$. The energy conservation law for the modulated REB following from the set of equations (20)-(22) has the form:

$$
\begin{aligned}
& \left(\nu_{b} / 2 \pi\right) \int_{0}^{2 \pi}\left(1+q^{2}\right)^{1 / 2} \Phi\left(\theta_{0}\right) d \theta_{0}+0.5|A|^{2}+2\left(\nu_{b} / 2 \pi\right)^{2} \gamma_{0}^{4} \lambda_{1}^{2} \Lambda_{1} \times \\
& \times\left\{\left[\int_{0}^{2 \pi} \Phi\left(\theta_{0}\right) \cos \theta d \theta_{0}\right]^{2}+\left[\int_{0}^{2 \pi} \Phi\left(\theta_{0}\right) \sin \theta d \theta_{0}\right]^{2}\right\}=\text { const. }
\end{aligned}
$$

Here the first term determines the energy contribution of the beam, and the second and third ones are the contributions of the amplified resonance wave and nonresonant harmonios into the energy balance of the system. From (1.2) we obtain the efficiency of exciting RF Pields in the waveguide:

$$
\begin{aligned}
& \eta=\frac{1}{2 \pi} \int_{0}^{2 \pi} \frac{\gamma_{0}-\left(1+q^{2}\right)^{1 / 2} \Phi\left(\theta_{0}\right)}{\gamma_{0}-1} d \theta_{0}=\eta_{1}+\eta_{2}=\frac{|A|^{2}-\left|A_{0}\right|^{2}}{2 \nu_{b}\left(\gamma_{0}-1\right)}+ \\
& +\frac{\nu_{b} \gamma_{0}^{4} \lambda_{1} \Lambda_{1}}{2 \pi^{2}\left(\gamma_{0}-1\right)}\left[\left[\int_{0}^{2 \pi} \Phi\left(\theta_{0}\right) \cos \theta d \theta_{0}\right]^{2}+\left[\int_{0}^{2 \pi} \Phi\left(\theta_{0}\right) \sin \theta d \theta_{0}\right]^{2}-(\pi g)^{2}\right\},
\end{aligned}
$$

where $\eta$ is the relative portion of total losses of REB kinetic energy, $\eta_{1}, \eta_{2}$ are the relative portions of beam energy losses due to the excitation of the resonant plasma waves and nonresonant harmonics of the plasma waveguide.

Let us consider briefly the linear stage of exciting plasma waves with the modulated REB. At the initial stage of the instability when the amplitude of the resonant plasma wave is small, the displacement 
and deformation of injected bunches in collective electromagnetio fields don't play the essential role. Therefore the driving beam may be regarded as the given modulated current. In the approximation of a given current we neglect the perturbed quantities compared with their equilibrium ones $\left(\theta_{0}, q_{0}\right)$. Then, as it follows from excitation equation (20), the amplitude of the resonant wave grows linearly $|A|=v_{b} g \xi / 2$. This occurs because already formed bunches enter the waveguide. Iinear growth of the field is a consequence of the resonance between the extermal force (modulated REB) and the plasma wave.

The processes of exciting RF fields by the modulated strong current REB and of accelerating the electrons of the driven beam by these fields are treated by simulation of Eqs.(20)-(24). Let us give the results of such modeling for the followi.ng parameters of the high-current REB: $\gamma_{0}=5 ; r_{1}=0.65 r_{0}(K=2) ; g=0.5 ; v_{b} / 2 \pi=3.4 \cdot 10^{-4}$ $\left(I_{b}=34 \mathrm{kA}\right)$. The parameters of the driven REB were as follows: $\gamma_{0}^{200}=5$; $r_{2}=0.1 r_{0}$. The boundary condition for the amplified plasma wave was taken the same as for the non-modulated driving REB: ReA $=5.10^{-3}$; $I_{m A}=0$. The value of the driving RFB current $I_{b}=34 \mathrm{kA}$ for the system's parameters given is close to the limiting current $I_{b}^{*}$ (see the intermediate report, section 4 ). Such a choice of $I_{b}$ permits to reveal the strong current effects during beam-plasma interaction as well as to obtain the limiting admissible strengths of accelerating fields in the considered approximation of the linear plasma.

Let us dwell, first, on the excitation of the plasma waveguide by the high-current REB. Fig.1 shows the amplitude $|A(\xi)|$ and the phase Arg $A(\xi)$ of the wave field in resonance with the beam versus the longitudinal coordinate $\xi$. The same Figure shows also the similar dependences obtained in the absence of the RF space oharge of the high-current $\mathrm{REBB}$. In the initial region of the interaction space the wave amplitude attains the maximum value at a distance considerably less than one for the unmodulated REB $\xi \cong 90$ (compare with the results given in the intermediate report). The linear growth of the amplitude is followed by trapping oscillations. Comparison of dependences depicted in the Figure shows that the account of RF space charge rield changes the plasma-beam instability saturation considerably: the magnitude of amplitude osoillations decreases and at $\xi>700$ the amplitude of the plasma wave assumes a constant value. 
This is the result of partial destruction of bunches of charged particles and of their phase mixing. The maximum of total energy Iosses of the high-current REB is achieved at the point $\xi \approx 90$ (the region of the first maximum of the amplitude) and it is equal to $\eta \approx 27 \%$.

Fig.2 shows the dependenoes of the field amplitude $|\tilde{A}(\xi)|$ and phase Arg $\tilde{A}(\xi)$ in the region of the driven REB $\left(r=r_{2}\right)$. At $\xi=0$ the field amplitude equals $|\widetilde{A}|=0.072$. It exoeeds considerably the amplitude of the amplified signal applied across the entrance in the region of the driven beam $\left(\left|A_{0}\right| K=0.01\right)$. This difference is due to the contribution into the resulting strength of the accelerating field at the entrance to the plasma waveguide made by the second term in (23) that is nonvanishing for the modulated REB at $\xi=0$. Thus in contrast to the non-modulated REBB case the acoelerated particles get into the field with a sufficiently large amplitude already at $\xi=0$.

On increasing $\xi$ the amplitude $|\tilde{A}|$ growth is changed by irregular space oscillations. The maximum value of the field amplitude in the region of the driven $\mathrm{REB}\left|\tilde{A}_{\max }\right| \approx 0.44$ is achieved at the point $\xi \approx 90$. This corresponds to the distance to the first maximum of the amplitude of the resonant plasma wave (see Fig.1a). At $\xi>90$ the amplitude falls and its value does not exceed $|\tilde{A}|=0.26$. Comparison of dependenoes shown in Figs.1a and $2 a$ shows that the field of the RF space charge of the driving REB enhances considerably the accelerating field amplitude over all length of the plasma waveguide. Thus, e.g., the value of $\left|\tilde{A}_{\max }\right|$ is approximately 4 times bigger than the maximum amplitude of the resonance wave in the region of the driven $R$ FB $\left|A_{\max }\right| \mathrm{K}=0.12$.

One oan draw the following conclusions from the results given.

a) The preliminary modulation of the high-current $R E B$ reduces substantially the distance to the first maximum of the accelerating field amplitude.

b) RF space charge field of the high-current modulated RFB increases the field amplitude in the region of the driven beam over all length of the accelerating gap. Already at $\xi=0$ the eleotrons of the driven beam get into the accelerating field exceeding oonsiderably the field of the resonant plasma wave.

Therefore the preliminary modulation of the high-current REB may 
play an important role in enhancing the efficiency of acceleration of driven beam electrons.

Consider now the acoeleration process of the driven beam eleotrons. The optimum length of the wareguide 1 at which the most effioient increase of eleotion energy oocurs has been determined according to the teohnique described in the intermediate report. This length is $1=96$ for the parameters considered. Fig. 3 depiots the electron momenta of the driven beam vs their initial phases $\theta_{0}^{a c o}$ at various points of the acoelerating gap. Already at the beginning of the accelerating gap the bunches of accelerated and decelerated partioles are formed. At the exit the maximum energy of acoelerated particles $W_{\max }=m c^{2}\left(\gamma_{\max }^{a 00}-1\right), \gamma_{\max }^{a 00}=\left[\left(q_{\max }^{a 00}\right)^{2}+1\right]^{1 / 2}$ exoeeds 4 times their initial energy. The number of acoelerated particles in the bunch at the exit of the wareguide amounts to ca. 17\% of the total number of particles (Fig.3d). The energy increase of the bulk eleotrons belonging to the aocelerated bunch possesses the markedly monotonous pattern. Fig.4 gives the good illustration of this picturing the dependence of the accelerated particle momentum with the initial phase $\theta_{0}^{a c c}=\pi$ versus the longitudinal coordinate $\xi$.

Now we present the estimates of the maximum strength of the longitudinal component of the electrio field in the driven beam region and of the electron acceleration rate. Plasma density is taken to be $n_{p}=10^{14} \mathrm{~cm}^{-1}$. For the driving REB parameters given above the maximum strength of the accelerating field is equal to (see Fig.2a): $\left|E_{\max }\right|=\left|\tilde{A}_{\max }\right| m c^{2} \omega / e \nabla_{0}=0.44 m c^{2} \omega / e \nabla_{0}$. As was shown in the intermediate report, the resonance frequency $\omega$ is close to the plasma irequency $\omega_{p}$. Then the maximum amplitude of the accelerating field is equal to $\left|E_{\max }\right|=420 \mathrm{Mer} / \mathrm{m}$. The length of the plasma waveguide equals $L=96 \nabla_{0} / \omega_{p} \approx 5 \mathrm{~cm}$, correspondingly. At this length the maximum inorease of energy, as it follows from Figs.3,4 amounts to $\Delta W=m c^{2}\left(\gamma_{\max }^{a c c}-\gamma_{0}^{a c c}\right) \simeq 5.8 \mathrm{MeV}$. Thus the acceleration rate is $\mathrm{T}=116 \mathrm{Mev} / \mathrm{m}$. The estimates given show that the modulation of the driving REB involves a remarkable increase of the acceleration rate (compare with the similar estimates given in the intermediate report). The increase of the acceleration rate is due first of all, to changes in the field amplitude distribution over the accelerating gap, as a result of which accelerated electrons are in the 
field of a sufficient amplitude already at $\xi=0$. Besides, in contrast to the non-modulated REB, the delayed exponential growth of the field amplitude to the first maximum is absent. These factors help to enhance considerably the acceleration rate of electrons without increasing the maximum strength of the accelerating field.

In conclusion, it should be noted the following. The above given values of the highest accelerating field intensity and the aoceleration rate of driven beam electrons were caloulated for the case of a homogeneous-density plasma. However, it is known that plasma inhomogeneity, which does exist in real plasma systems, may cause violation of synchronism between the beam and the plasma wave due to the change in the phase velocity of the wave. This may be one of the essential factors that impede the effective plasma-wave excitation and hence, the electron acceleration. By way of example we shall give the requirements to the inhomogeneity scale for the linear plasma density profile

$$
n_{p}(z)=n_{p o}\left(1+z / L_{p}\right)
$$

$I_{p}$ is the characteristic length of inhomogeneity; $n_{p o}=n_{p}(z=0)$. For this purpose we shall derive the expression to describe the variation of the longitudinal wave number $h_{1}$, belonging to the resonant plasma wave, as a function of the coordinate $z$ :

$\Xi(z) \equiv 1-h_{1}(z) / h_{1}(z=0), \quad h_{1}(z=0)=\omega / \nabla_{0}$.

Using the expression for the dispersion of the cylindrical plasma waveguide of radius $r_{0}$, located in a strong longitudinal magnetic field

$$
\begin{aligned}
& \left(\lambda_{1} / r_{0}\right)^{2}=\left[(\omega / c)^{2}-n_{1}^{2}\right]\left(1-\omega_{p}^{2} / \omega^{2}\right), \\
& \lambda_{1}=2.405, \omega_{p}=\left(4 \pi n_{p} e^{2} / m\right)^{1 / 2},
\end{aligned}
$$

and taking into account (1.4), we obtain the expression for $\Xi(z)$ :

$$
\Xi(z)=0.5 \frac{\omega_{p}^{2}(z=0) r_{\circ}^{2} z}{\gamma_{0}^{4} \lambda_{1}^{2} \beta_{o}^{2} c^{2} L_{p}} .
$$

It is evident that at a fixed frequency of the enhanced plasma wave $\omega$, expression (1.6) describes the variation of the phase velocity of the plasma wave relative to the beam velocity: $\Xi(z) \equiv 1-h_{1}(z) / h_{1}(z=0)=$ $=\left(\nabla_{0}-\nabla_{p h}\right) / \nabla_{0}, \nabla_{p h}=\omega / h_{1}$. For a premodulated beam the breakdown of 
plasma-beam instability occurs at such a plasma inhomogeneity, when the phase difference between the beam and the wave gained over the length of interaction is equal to $\pi$. Hence we come to the inequality defining the admissible values of plasma inhomogeneity length, at which no breakdown of plasma wave exoitation take place:

$$
I_{p}>\frac{\omega_{p}^{3}(z=0) r_{0}^{2} I_{1}^{2}}{4 \pi \gamma_{0}^{4} \nabla_{0}^{3} \lambda_{1}^{2}}
$$

For the above given parameters of the system and $r_{0}=20 m$, it follows from (1.7) that $I_{p}>14 \mathrm{om}$. So, the distanoe orer whioh the plasma density is doubled at the expense of inhomogeneity must be essentially greater than the length of the acoelerating gap.

2. Two-beam acceleration of charged particles in a corrugated waveguide

2.1. Statement of the problem. Input equations.

Let us consider a segment of length $I$ of a corrugated waveguide with an ideally conduoting lateral surface. The radius of the waveguide walls varies in acoordance with the harmonic law:

$$
r(z)=r_{0}\left(1+\alpha \cdot \operatorname{cosk}_{0} z\right) \text {, }
$$

where $k_{0}=2 \pi / D, D$ being the waveguide spatial period, $r_{0}$ is the average radius of the waveguide, $\alpha=\Delta r / r_{0} \ll 1$ is the periodical inhomogeneity parameter of the electrodynamical structure, $\Delta \mathrm{r}$ is the corrugation depth. The waveguide is completely consistent on both sides (refleoted waves are absent). Along the z-axis of the waveguide two tubular monoenergetio REB propagate (Fig.5). The radii of the driving beam and the driven one are respeotively $r_{1}$ and $r_{2}$ $\left(r_{1}<r_{2} \propto r_{0}\right)$.

In the acoeleration soheme under consideration, the driving highcurrent REB of a relatively low initial energy $w_{0}=m^{2}\left(\gamma_{0}-1\right)\left(\gamma_{0}\right) 1$ is the relativistio factor of the driving beam, $m$ is the eleotron mass, $c$ is the speed of light), which excites the eleotromagnetic field, is in the Cherenkor resonance with the fundamental radial mode $(s=1)$ of the first space harmonio $(n=1)$ of the backward wave: $\omega_{0}=\left[k_{0}-h_{1}\left(\omega_{0}\right)\right] \nabla_{0}$, where $\nabla_{0}$ is the initial driving REB velooity, $\omega_{0}$ 
is the resonance frequency of the wave, $h_{1}$ is the longitudinal wave number (the subscript "1" denotes the radial mode number). The driven beam is injected from the exit side of the waveguide ( $(=L)$ with the initial energy $W_{0}^{a 00}=m c^{2}\left(\gamma_{0}^{a c o}-1\right), \gamma_{0}^{a 0 c}>\gamma_{0}\left(\gamma_{0}^{a c 0}\right.$ is the relativistio lactor of the driven beam) and is in the Cherenkor resonanoe with the zero spaoe harmonio of the baokward wave $(n=0): \omega_{0}=h_{1} \nabla_{0}^{a c c}$, $\nabla_{0}^{a 00}$ being the initial valooity of the driven REB (Fig.6). The zero and the first space harmonios are parametrioally interrelated by a spatial period of the structure, $D$.

The physioal model adopted here to desoribe the processes of $\mathrm{RF}$ field exoitation by a high-current REB and the acoeleration of driven beam eleotrons by these fields involves the following statements:

1. The eleotrodynamio struoture under study is in a strong longitudinal magnetio lield, therefore there is no transverse displacement of beam eleotrons (the motion is one dimensional).

2. The beams are infinitely thin, therefore their stratifioation is negleoted.

3. Assuming the driven beam ourrent to be small in comparison with the driving beam ourrent, we shall neglect its influenoe on the RF field generation in the corrugated waveguide. So, the aocelerated eleotrons move in the given eleotromagnetic field.

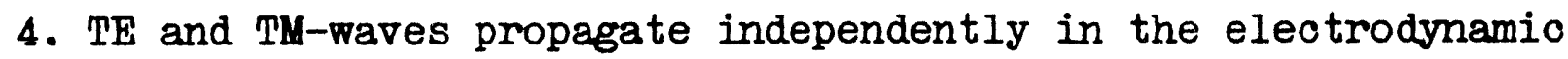
structure under study. Sinoe only TM waves perturb the longitudinal component of the beam velooity as well as the beam density, we shall consider below the excitation of this type of the waves.

The original set of equations describing the RF field excitation by a high-current in a corrugated waveguide comprises the Maxwell's equations written in the oylindrical coordinate system:

$$
\begin{aligned}
& \frac{\partial \mathrm{E}_{\mathrm{r}}}{\partial \mathrm{z}}-\frac{\partial \mathrm{E}_{\mathrm{z}}}{\partial \mathrm{r}}=-\frac{1}{\mathrm{c}} \frac{\partial \mathrm{H}_{\varphi}}{\partial \mathrm{t}}, \\
& \frac{\partial \mathrm{H}}{\partial \mathrm{z}}=-\frac{1}{\mathrm{c}} \frac{\partial \mathrm{E}_{\mathrm{r}}}{\partial \mathrm{t}}, \\
& \frac{1}{\mathrm{r}} \frac{\partial}{\partial \mathrm{r}}\left(\mathrm{rH}_{\varphi}\right)=\frac{1}{\mathrm{c}} \frac{\partial \mathrm{E}_{\mathrm{z}}}{\partial \mathrm{t}}+\frac{4 \pi}{\mathrm{c}} \mathrm{J}_{\mathrm{b}},
\end{aligned}
$$

where $E_{Z}, E_{r}, H_{\varphi}$ are the components of the TM-wave electric and mag- 
netio fields, $J_{b}$ is the diving REB ourrent density. To solve equations (2.2)-(2.4), we make use of the Fourier-series expansion of the variables which enter into these equations.

$$
\begin{gathered}
\vec{E}=\operatorname{Re} \int_{0}^{\infty} \vec{E}_{\omega} \exp (-1 \omega t) d \omega, \overrightarrow{\mathrm{H}}=\operatorname{Re} \int_{0}^{\infty} \overrightarrow{\mathrm{H}}_{\omega} \exp (-1 \omega t) d \omega, \\
J_{b}=\operatorname{Re} \int_{0}^{\infty} J_{\omega} \exp (-1 \omega t) d \omega
\end{gathered}
$$

Then, for the complex-valued variables $E_{r \omega}, E_{z \omega}, H_{\varphi \omega}, J_{\omega}$ we obtain the lollowing equations:

$$
\begin{aligned}
& \frac{\partial E_{r \omega}}{\partial z}-\frac{\partial E_{z \omega}}{\partial r}=1-\frac{\omega}{c} H_{\varphi \omega}, \\
& \frac{\partial H_{\varphi \omega}}{\partial z}=1 \frac{\omega}{c} E_{r \omega}, \\
& \frac{1}{r} \frac{\partial}{\partial r}\left(r H_{\varphi \omega}\right)=-\frac{\omega}{c} E_{z \omega}+\frac{4 \pi}{c} J_{\omega} .
\end{aligned}
$$

According to the general theory of waveguide exoitation [7], the expressions for the fields entering into $(2.6)-(2.8)$ oan be written as

$$
\begin{aligned}
& \overrightarrow{\mathrm{E}}_{\omega}=\sum_{s=1}^{\infty}\left(C_{s \omega} \overrightarrow{\mathrm{E}}_{s}+C_{-s \omega} \overrightarrow{\mathrm{E}}_{-s}\right)+\frac{4 \pi}{1 \omega} j_{\omega} \vec{e}_{z}, \\
& \overrightarrow{\mathrm{H}}_{\omega}=\sum_{s=1}^{\infty}\left(C_{s \omega} \overrightarrow{\mathrm{H}}_{s}+C_{-s \omega} \overrightarrow{\mathrm{H}}_{-s}\right),
\end{aligned}
$$

where $\vec{E}_{ \pm s}, \vec{H}_{ \pm s}$ are the eigenwave fields of the eleotrodynamio structure without a beam $\left(\vec{E}_{-s}=\vec{E}_{s}^{*}, \vec{H}_{s}=-\vec{H}_{s}^{*}\right), C_{ \pm s w}$ are their amplitudes. The summation in (2.9), (2.10) is taken over all radial modes of the wareguide. The indioes $s$ and $-s$ refer to direot and backward waves, respeotively.

The amplitudes $\mathrm{C}_{ \pm s \omega}$ satisfy the following equations of exoitation:

$$
\frac{d C}{d z} \pm s \omega= \pm \frac{1}{N_{s}} \int_{s} J_{\omega} E_{\mp s z} d s_{1},
$$

where $N_{s}=\frac{c}{4 \pi} \int_{s_{1}}\left\{\left[\vec{E}_{s} \times \vec{H}_{-s}\right]-\left[\vec{E}_{-s} \times \vec{H}_{s}\right]\right\} \vec{e}_{z} d s_{1}$ is the norm of the $s-t h$ radial mode, $s_{1}$ is the waveguide oross section.

Assuming that we have the exoitation of a narrow warepacket with 
the frequenoy interval $2 \Delta \omega$ and the average irequency $\omega_{0}\left(\omega=\omega_{0} \pm \Delta \omega\right.$, $\left.\Delta \omega / \omega_{0} \ll 1\right)$, and employing the inverse Fourier transformation for (2.11) we obtain the equations which describe the space-time variation of the fields of direct and counter backward waves in the electrodynamio structure:

$$
\frac{1}{\nabla_{g s}} \frac{\partial C_{ \pm s}}{\partial t} \pm \frac{\partial C_{ \pm s}}{\partial z}=\frac{2}{N_{s} T_{0}} \int_{s_{1}} d s_{\perp} \int_{t-T_{0} / 2}^{t+T_{0} / 2} j_{b s z} E_{F s p}\left(1 \omega_{0} t^{\prime}\right) d t^{\prime},
$$

where $C_{ \pm s}(z, t)=\int_{-\Delta \omega^{\prime}}^{\Delta \omega} C_{ \pm s} \exp \left[ \pm i\left(\Delta \omega^{\prime} / \nabla_{g s}\right) z-1 \Delta \omega^{\prime} t\right] d \Delta \omega^{\prime}, T_{0}=2 \pi / \omega_{0}$, $\nabla_{g s} \equiv 1 /\left(d h_{s} / d \omega\right)$ is the group velosity of waves; the $\nabla_{g s}, N_{s}, E_{\text {fsz }}$

values are taken at the Irequency $\omega_{0}$. Note, that by separating the narrow Irequency interval $\Delta \omega / \omega_{0} \ll 1$, we impose thereby the requirement on the slow variation of the functions $C_{ \pm s}(z, t):\left|\left(\partial C_{ \pm s} / \partial t\right) T_{0} / C_{ \pm s}\right| \ll 1$.

To obtain the self-consistent set of equations desoribing the processes of RF field generation by a diving REB one must use the kinetio vlasor equation for the electron distribution function:

$$
\frac{\partial \rho}{\partial t}+\nabla \frac{\partial f}{\partial z}-e E_{z} \frac{\partial \rho}{\partial p}=0
$$

with a boundary condition at the interaction space input

$$
f(t, p, r, z=0)=f_{0}(t, p, r) \text {, }
$$

where $e$ is the charge of the electron; $\nabla, p$ are, respectively, the velooity and momentum of electrons, $f$ is their distribution function. The beam current density is given by

$$
J_{b}=-e \int \rho \nabla d p \text {. }
$$

The problem of integrating the kinetic equation (2.13) is equivalent to the integration of the corresponding oharacteristic set of equations

$$
\begin{aligned}
& \frac{d t}{d z}=\frac{1}{\nabla}, \\
& \frac{d p}{d z}=-\frac{e}{m} E_{z},
\end{aligned}
$$

the solution of which can be represented as 


$$
t=t_{l}\left(t_{0}, p_{0}, z\right), p=p_{l}\left(t_{0}, p_{0}, z\right),
$$

where $t_{0}$ is the time of electron entrance into the waveguide, $p_{0}$ is the initial electron momentum value. Note, that expression (2.18) describe the motion of beam particles along the Lagrange trajeotories.

Making use of the law of phase volume conservation on the Lagrange trajectories of beam particles we represent the desired distribution function 1 in the following form:

$$
\begin{aligned}
& I(t, p, r, z)=\int d t_{0} \int d p_{0} f_{0}\left(t_{0}, p_{0}, r\right) \delta\left[t-t_{l}\left(t_{0}, p_{0}, z\right)\right] . \\
& . \delta\left[p-p_{l}\left(t_{0}, p_{0}, z\right)\right] \nabla_{0} / \nabla
\end{aligned}
$$

Here $f_{0}\left(t_{0}, p_{0}, r\right)=N_{b} \delta\left(r-r_{1}\right) \delta\left(p_{0}-P_{0}\right) \Phi\left(t_{0}\right) / 2 \pi r_{1}$ is the initial distribution function of electrons; $N_{b}$ is the particle density per unit length; $\delta(x)$ is the Dirac function, $\Phi\left(t_{0}\right)$ is the distribution funotion of eleotrons in initial times of their entrance into the waveguide. Further on, we assume that a non-modulated high-ourrent REB is injected into the waveguide $\Phi\left(t_{0}\right)=1$. Substituting (2.15) into (2.12) and taking into account expression (2.19) we obtain the following equations for $C_{ \pm s}(z, t)$ :

$$
\frac{1}{\nabla_{g s}} \frac{\partial C_{ \pm s}}{\partial t} \pm \frac{\partial C_{ \pm s}}{\partial z}=-\frac{2 I_{b}}{N_{s} T_{0}} \int r d r \int_{X(t, z)} \delta\left(r-r_{1}\right) r_{1}^{-1} E_{\mp s z} \exp \left(1 \omega_{0} t_{l}\right) d t_{0},(2.20)
$$

where $I_{b}=e N_{b} \nabla_{0}$ is the beam current. Limits of integration of $X(t, z)$ in $t_{0}$ follow from the solution of the functional equation:

$$
t_{l}\left(t_{0}, p_{0}, z\right)=t \pm T_{0} / 2
$$

In the approximation of a small change in the beam particle velocity during interaction with resonance wave (this approximation is valid for Cherenkov oscillators with an inertial mechanism of beam particle grouping), the limits of integration in to are substantialIy simplified and can be represented explicitly. To this approximation the $t_{l}$ value can be defined as: $t_{l}=t_{0}+z / \nabla_{0}+\Delta t_{l}\left(\Delta t_{l} \ll z / \nabla_{0}\right)$. Neglecting $\Delta t_{l}$ and replacing $t$ by $t-z / \nabla_{0}$, we obtain the following relationship for the limits of integration:

$$
t-T_{0} / 2 \leqslant t_{0} \leqslant t+T_{0} / 2 \text {. }
$$

In view of substitution made, Eqs. (2.20) take on the form: 


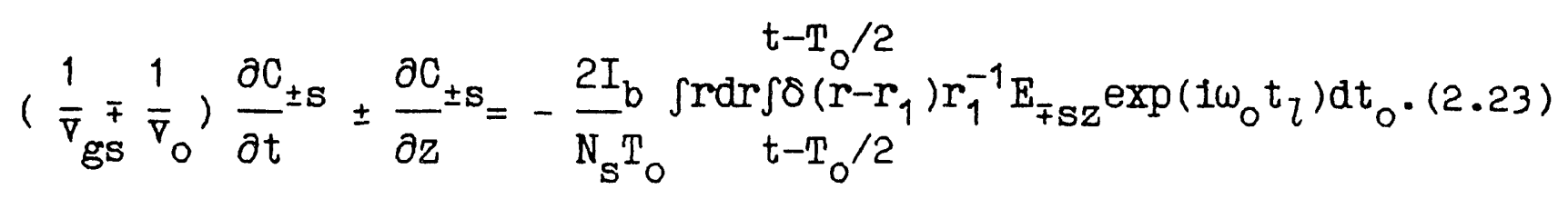

Eqs. (2.16), (2.17), (2.23) are a self-consistent set of equations describing the dynamios of RF field excitation by the driving REB in the electrodynamic structure. For further analysis of the equations derived, it is necessary to define explioitly the function $E_{z}$. For this purpose, acoording to Floquet's theorem, we represent the eigenfields of the periodic structure under study as space harmonic series:

$$
E_{ \pm s z}=\sum_{n=-\infty}^{\infty} a_{n} F_{s n}(r) \exp \left[1\left( \pm h_{s}+k_{0} n\right) z\right], a_{0}=1
$$

where $a_{n}$ is the $n$-th space harmonic amplitude, $F_{s n}$ is the function describing the radial field distribution in the waveguide.

It will be reoalled that the driving REB is in the Cherenkor resonance with the first space harmonic $(n=1)$ of the fundamental radial mode $(s=1)$ of the backward wave. As to the nonresonant terms, they contribute, together with the beam term in (2.9), to the RF space charge field. Since the space harmonic amplitudes $a_{n}$ deorease as $a^{n}$ with their growing number [4], we shall restrict ourselves to considering only the nonresonant terms of the zero order in $a$. Hence, the resultant expression for the longitudinal electric field component in the region of the driving REB can be written as

$$
\begin{aligned}
& E_{z}\left(r_{1}, z, t\right)=\operatorname{Re}\left\{\left[C_{-1}(t, z) I_{0}\left(x r_{1}\right) a_{1}+\frac{21 \omega_{0} I_{b} G \quad t+T_{0} / 2}{\pi v_{0}^{2} \gamma_{0}^{2}} \int \exp _{t-T_{0} / 2}\left[1 \omega_{0} t_{l}-1\left(-h_{1}+k_{0}\right) z\right] .\right.\right. \\
& \cdot \exp \left[-1 \omega_{0} t_{\imath}+1\left(k_{0}-h_{1}\right) z\right],
\end{aligned}
$$

here $x=\left(-h_{1}+k_{0}\right) / \gamma_{0} ; G=\left[K_{0}\left(x r_{1}\right) I_{0}\left(x r_{0}\right)-K_{0}\left(x r_{0}\right) I_{0}\left(x r_{1}\right)\right] I_{0}\left(x r_{1}\right) / I_{0}\left(x r_{0}\right)$; $I_{0}$ is the zero-order modified Bessel function, $K_{0}$ is the zero-order Modonald funotion. The second term in (2.25) describes the RF space charge field of a high-ourrent $\mathrm{REB}, \mathrm{C}_{-1}(t, z)$ is a slowly varying complex amplitude of the resonating backward wave which satisfies Eq. (2.23). As a result, the self-consistent set of equations describing the $R F$ field generation can be represented by: 


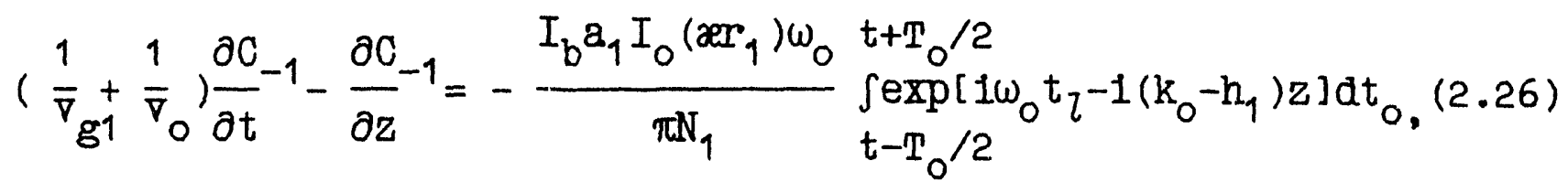

$$
\begin{aligned}
& \frac{d t_{l}}{d z}=\left(p_{\imath}^{2}+m^{2} c^{2}\right)^{1 / 2}\left(p_{l} c\right)^{-1} \\
& \frac{d p_{l}}{d z}=-e\left(p_{\imath}^{2}+m^{2} c^{2}\right)^{1 / 2}\left(p_{l} c\right)^{-1} E_{z}\left(r_{1}, z, t\right) .
\end{aligned}
$$

For lurther analysis, it appears more convenient to write this set of equations in a dimensionless form:

$$
\begin{aligned}
& \frac{\partial A_{-}}{\partial \tau}-\beta_{-} \frac{\partial A_{-}}{\partial \xi}=v_{b_{\tau}} \int_{\tau-\pi}^{\tau+\pi} \exp (1 \theta) d \theta_{0}, \\
& \frac{d \theta}{d \xi}=\beta_{0}\left(1+q^{-2}\right)^{1 / 2}-1, \\
& \frac{d q}{d \xi}=-\operatorname{Re}\left\{\left[A_{-}+10 \int_{\tau-\pi}^{\tau+\pi} \exp (1 \theta) d \theta_{0}\right] \exp (-1 \theta)\right\}\left(1+q^{-2}\right)^{1 / 2},
\end{aligned}
$$

where $A_{-}=C_{-1} e a_{1} I_{0}\left(2 r_{1}\right)\left[m c^{2}\left(k_{0}-h_{1}\right)\right]^{-1}, \tau=\omega_{0}\left(t-z / \nabla_{0}\right)$, $\xi=\left(k_{0}-h_{1}\right) z, \beta_{0}=\nabla_{0} / c, \beta_{-}=\beta_{g 1} /\left(1+\beta_{g 1}\right), \beta_{g 1}=\nabla_{g 1} / \nabla_{0}, \vartheta=\omega_{0} t_{\tau}-\left(k_{0}-h_{1}\right) z$, $\theta_{0}=\theta(\xi=0), q=p_{l} /$ mc is the dimensionless momentum of the electrons, $\sigma=\frac{2 I_{b} G}{\pi I_{A} \beta_{0} \gamma_{0}^{2}}, I_{A}=m c^{3} / e \simeq 17 k A, \nu_{b}=\frac{2 I_{b}\left[\lambda_{1} a_{1} I_{0}\left(x r_{1}\right)\right]}{\pi I_{A} \beta_{O}^{3} \gamma_{O}^{4}\left(x r_{0}\right)^{4} J_{1}^{2}\left(\lambda_{1}\right)\left(1+\beta_{g_{1}}\right)}$, $J_{1}$ is the first-order Bessel function, $\lambda_{1}=2.405$ is the first root of the zero-order Bessel function. Note that for writing $\mathrm{N}_{1}$ we made use of the expression for the smooth waveguide. This is valid for a small value of the periodic inhomogeneity $\alpha \ll 1$, so we have: $N_{1}=-\nabla_{g 1} \omega_{o}^{2} r_{o}^{4} J_{1}^{2}\left(\lambda_{1}\right) /\left(2 \lambda_{1}^{2} c^{2}\right)$.

For the given set of equations in the case of steady-state regime of generation ( $A_{-}$is time independent), we can write the first integral

$$
v_{b} \int_{-\pi}^{\pi}\left(1+q^{2}\right)^{1 / 2} d \theta_{0}-0.5 \beta_{-}\left|A_{-}\right|^{2}=\text { const }
$$


which is the law of energy conservation.

The effioienoy of transformation of the beam energy into the eleotromagnetic wave energy is defined by the expression:

$$
\eta=\frac{S(z=0)-S(z=L)}{S(z=0)},
$$

with $S(z)$ being the beam energy IIux, which can be rewritten as

$$
\eta=\frac{1}{2 \pi} \int_{-\pi}^{\pi}\left[\gamma_{0}-\left(1+q^{2}\right)^{1 / 2}\right]\left(\gamma_{0}-1\right)^{-1} d \theta_{0}
$$

The equations desoribing the motion of the driven beam electrons in the RFB-exoited lield have the forms:

$$
\begin{aligned}
& \frac{d \theta^{a c c}}{d \xi^{\prime}}=\beta_{o}\left[1+\left(q^{a c c}\right)^{-2}\right]^{1 / 2}-\beta_{o} / \beta_{0}^{a c c}, \\
& \frac{d q^{a c c}}{d \xi^{\prime}}=\operatorname{Re}\left[A_{-} \exp \left(-1 \theta^{a c c}\right)\right]\left[1+\left(q^{a c o}\right)^{-2}\right]^{1 / 2} R,
\end{aligned}
$$

where $\xi^{\prime}=(I-z)\left(k_{0}-h_{1}\right), q^{a c 0}=p_{l}^{a c 0} / m c$ is the dimensionless momentum of the driven beam electrons, $\beta_{0}^{a 0 c}=\nabla_{0}^{a 00} / c, \quad \theta^{a 00}=\omega_{0} t_{l}^{a 00}+h_{1}(L-z), t_{l}^{a 00}$ is the time of the driven beam electron arrival at the point with the coordinate $\mathrm{I}-\mathrm{Z}$,

$\mathrm{R}=\frac{1}{a_{1}} \frac{I_{0}\left(h_{1} r_{2} / \gamma_{0}^{a 00}\right)}{I_{0}\left[\left(k_{0}-h_{1}\right) r_{1} / \gamma_{0}\right]}$ is the parameter defining the ratio of the fundamental space harmonic amplitude $a_{0}=1$ in the driven beam region to the lirst space harmonic amplitude $a_{1}$ in the driving beam region. Note that the $R$ value is determined not only by the ratio of space harmonic amplitudes but also by the ratio of beams radii. The efficienoy of the energy transfer from the high-current REB to accelerated eleotrons is dependent on this parameter. In writing (2.35), (2.36) we have used the new variables $2^{\prime} \rightarrow L-z, p_{l}^{a c c} \rightarrow-p_{l}^{a c c}$, which are convenient for describing the process of relativistic electron acceleration.

The set of equations derived must be complemented with initial and boundary conditions for the field and electrons of the beams:

$$
\begin{aligned}
& A_{-}(\tau=0, \xi)=\tilde{A}, A_{-}(\tau, \xi=\tilde{L})=0, \tilde{I}=L\left(k_{0}-h_{1}\right), \\
& q(\xi=0)=q_{0}=\beta_{0} \gamma_{0}, q^{a c c}\left(\xi^{\prime}=0\right)=q_{0}^{a c c}=\beta_{0}^{a c o} \gamma_{0}^{a c c},
\end{aligned}
$$


$\tilde{A} \theta(\xi=0)=\theta^{a c c}\left(\xi^{\prime}=0\right) \in[\tau-\pi, \tau+\pi]$,

where $\tilde{A}$ is the initial electric field perturbation. Note, that the above given boundary and initial conditions for the field correspond to the BWO without wave reflection on the waveguide ends.

\subsection{Result of numerioal simulations}

The investigation of the two-resonance soheme of acceleration in a corrugated waveguide was studied by numerioal simulation of the set of Eqs. (2.29)-(2.31), (2.35), (2.36) for the following initial beam energies $\gamma_{0}=3, \gamma_{0}^{a 0 c}=6$ and the initial electrio field perturbation $\operatorname{Re} \tilde{A}=5 \cdot 10^{-3}, \operatorname{Im} \tilde{A}=0$. The geometrical parameters of the slow wave structure were determined from the requirement of slowing down the phase velocity of the main space harmonio to $\gamma_{p h}=\gamma_{0}^{a 0 c}=6$. Aocording to [8], the correlation between the phase velooity of the zero space harmonic and the geometrical parameters of the waveguide is given by the expression:

$\gamma_{\mathrm{ph}}=\frac{\mathrm{x}}{\alpha^{1 / 2}}\left[(1+2 \alpha) /\left(\mathrm{x}^{2}-2\right)\right]^{1 / 2}, \mathrm{x}=a^{1 / 2} \pi \mathrm{r}_{0} / \mathrm{D}$. The parameter $\alpha$ was chosen equal $\alpha=8.5 \cdot 10^{-2}$. Then from the last expression we can find $r_{0} / D \simeq 1.6$. Taking this into account, we can find the amplitude of the first space harmonic $a_{1}$ and the group velocity of the wave as $a_{1} \simeq 0.28$, $\beta_{g 1} \approx 0.8$ respectively [4]. The values of the resonance wavenumbers $h_{1}\left(\omega_{0}\right), k_{0}-h_{1}\left(\omega_{0}\right)$ (Fig.6) were found from the condition of Cherenkov synchronism between the RFB and the slow backward wave $h_{1} \approx k_{0} / 2$ [9]. Further on, we consider the radius of the driven REB to be fixed $r_{2} / r_{0} \simeq 1$. The calculations were carried out for different values of the waveguide length $L$, driving current $I_{b}$ and radius $r_{1}$.

We Pirst consider the case, when $r_{1} / r_{0}=0.5 \quad(G=0.51, R=3.6)$. The space-charge limiting current $I_{s c l}$ for a thin hollow REB with this radius is equal to $I_{\text {scl }}=17 \mathrm{kA}\left(\gamma^{2 / 3}-1\right)^{3 / 2} /\left[2 \ln \left(r_{0} / r_{1}\right)\right] \approx 14 \mathrm{kA}$. The waveguide length is chosen to be $\tilde{I}=44$ from the condition for the onset of a steady-state harmonic of generation, which is close to the optimum in effioienoy $\gamma_{0}=3[10]$. For these parameters the oscillations get excited at $\nu_{b}=6.10^{-4}\left(I_{b} \approx 5 \mathrm{KA}\right)$ and the steady-state harmonic of generation sets in. Qualitatively, the excitation of stationary RF fields by a high-current REB can be described as 
follows (Fig.7). At the linear stage there is an exponential growth of the output-signal amplitude. At the same time the maximum amplitude value shifts from the output to the input of the waveguide at a velooity close to the group one. Then, after a typical amplitude peak in the vicinity of $\xi=0$ there ocour damped osoillations about stationary state. The process is completed by a steady-state rield distribution along the interaction space. Note, that the efficienoy of the stationary regime of generation (2.34) is about $\eta \approx 17 \%$.

As current $I_{b}$ approaches to the value corresponding to the limiting vacuum current $I_{s c l}$, the electric lield intensity inoreases (Fig.8). In this case, in the range of $v_{b}$ values considered the steady-state one-prequenoy mode of generation remains stable. The stabilj.ty of stationary states of the eleotrodynamio system at $\tilde{L}=44$ is due to the fact that the RF space charge field of the high-current REB prevents the nonlinear rearrangement of beam eleotrons, which is associated with arising nonstationary sel1modulation regimes of generation. A more detailed disoussion of this problem can be found in [11].

Let us now consider the process of charged particle acceleration by a monochromatic eleotromagnetic back-ward wave. Figs.9,10 show the phase planes of the driving beam particles $q^{200}, \varphi^{200}$, where $\varphi^{\mathrm{acc}}=\varphi-\vartheta^{\mathrm{acc}}, \varphi$ is the phase of the complex amplitude of the backward wave $A_{-}=\left|A_{-}\right| \exp (1 \varphi)$; and the dependences of eleotron momenta $q^{200}$ on the initial phase $\vartheta_{0}^{a c c}$ of eleotrons. It is seen from these figures, that already at the beginning of the interaction space the bunches of acoelerated and decelerated particles are formed. The accelerated eleotrons make up approximately $20 \%$ of the total amount of beam particles. For the parameters considered the maximum energy of the accelerated eleotrons is about three times as large as its initial value is about three times as large as its initial value $\mathrm{W}_{0}^{200}$. The relative energy soattering of the accelerated particle is no more than 10\%. As the numerical analysis shows, the phase difference for the particles belonging to the acoelerated bunch $\left(\Delta \varphi^{200}=\varphi^{\mathrm{acc}}\left(\xi^{\prime}=\tilde{L}\right)-\varphi^{20 c}\left(\xi^{\prime}=0\right)\right)$ is approximately equal to $\pi$ and throughout the length of the interaotion space $\theta^{\text {aco }}$ weakly changes its initial value $\theta_{0}^{a c c}$. Therefore, the electric field, which aots on the acoelerated electrons, ohanges its polarity only near $\xi^{\prime}=\tilde{\mathrm{L}}$. So, the energy gain of the partioles of the driven REB has mono- 
tonous character. This is illustrated by Fig.11, which shows the dependenoe of the eleotron momentum on the longitudinal coordinate $\xi^{\prime}$ of one of the particles belonging to the aocelerated bunch.

The deorease of the driving RFB radius $r_{1} / r_{0}=0.3 \quad(G=0.9, R \approx 4.1$, $I_{\mathrm{SOl}}=8 \mathrm{kA}$ ) does not essentially ohange the process of generation of RF eleotromagnetio fields. At the same time the inorease of the transformation parameter $R$ allows one to reach a greater acceleration rate in comparison with the oase of $r_{1} / r_{0}=0.5$. Fig.12 shows the maximum energy gain of the aocelerated particles $\Delta W^{a c 0}$ versus the driving beam current $I_{b}$. It is evident that in a wide interval of the driving beam currents an effective charged particle acceleration takes plaoe. Here we should note that at $r_{1} / r_{0} \rightarrow 0$ along with the growing transformation coeffioient $R$, the coupling between the driving beam and the resonance backward eleotromagnetic wave becomes weaker $\left(\nu_{b}\right.$ decreases $)$. Besides, the space-charge limiting beam ourrent value in the waveguide $I_{\text {sol }}$ also deoreases. So, further reduction of the driving beam radius $\left(r_{1} / r_{0}<0.3\right)$ seems unreasonable.

Now we present the estimates of the maximum longitudinal oomponent of the eleotric field and the acceleration rate which oan take place in the two-resonance acceleration scheme under consideration for the following parameters: $r_{1} / r_{0}=0.5 ; v_{b}=1.6 \cdot 10^{-3}\left(I_{b} \approx 13 \mathrm{kA}\right)$, $\mathrm{D}=1.2 \mathrm{om}$. For these parameters the maximum eleotrio field intensity in the region of driven REB (Fig.8) is: $\left|\mathrm{E}_{-}\right|_{\max } \propto 0.14 \mathrm{mc}^{2}\left(\mathrm{k}_{\mathrm{o}}-\mathrm{h}_{1}\right) \mathrm{R} / \mathrm{a}_{1} \mathrm{eI}\left(2 \mathrm{er} \mathrm{r}_{1}\right) \approx 190 \mathrm{Mv} / \mathrm{m}$. The length of the slow-wave structure for $\mathrm{D}=1.2 \mathrm{~cm}$ is equal, acoordingly, to $17 \mathrm{~cm}$. As it follows Fig.8, at this length the acceleration rate is about $\mathrm{T} \approx 35 \mathrm{Mev} / \mathrm{m}$. It should be stressed that significant difference between $\left|E_{-}\right|_{\max }$ and the acceleration rate $T$ is due to an inhomogeneous distribution of the longitudinal component of the electric field along the slow-wave struoture (Fig.8), and to the variation of electric field phase $\varphi$ during acceleration of charged particles.

The above given results were obtained at a fixed $\tilde{L}$ value, when a steady state regime of RF field generation was attained for the self-oscillation system. With an inoreasing waveguide length, the steady-state regime becomes unstable and ohanges into a self-modulation one [11]. The structure length value at which this regime sets in, depends on the initial REB energy and ourrent. For the self-modulation regime of generation the field distribution along 
the wareguide appears to be a sequence of amplitude |A_l peaks and dips of different height and shape. Numerical oaloulations have shown that this field distribution leads to a nonmonotone energy gain by driven beam electrons. As a result, the aoceleration rate falls down to $\mathrm{T}<35 \mathrm{Mev} / \mathrm{m}$. So, in the two-resonanoe acoeleration scheme considered the most effective aooeleration regime is acoomplished when a steady-state regime of generation is reached in. the system.

\section{Basic results. Conolusions}

In conclusion let us formulate the basic results obtained in this work.

In seotion $I$ of the work considers the aoceleration of electrons by the high-current RRB in the plasma waveguide.

1.1 The exoitation of aocelerating fields by the modulated highourrent REB in the plasma waveguide has been treated by numerical means. The preliminary modulation of the beam is shown to shorten the distance to the first maximum of the accelerating field amplitude. The RF space charge field is established to inorease the field amplitude in the region of the driven beam over all the aocelerating gap $(0 \leqslant \xi \leqslant 1)$.

1.2 A process of acoelerating the electrons of the driven beam is described. The estimates of the maximum strength of the aooelerating field and the acceleration rate for electrons are given. The preliminary modulation of the driving RABB is established to enhance the acoeleration rate of driven beam eleotrons.

1.3 The effeot of plasma inhomogeneity on the process of plasma wave exoitation has been considered. Admissible gradients of plasma inhomogeneity are found, at which there is no breakdown of plasma wave exoitation and acceleration of driven beam eleotrons.

In seotion II we have considered the two-beam scheme of charged partiole acoeleration in a vacuum corrugated waveguide.

2.1 In the framework of the space-time model the nonlinear set of equations has been derived to describe the process of eleotromagnetic wave generation by a high-ourrent REB. The set of equations has been analyzed by numerioal simulation.

2.2 The RF field exoitation by a high-ourrent REB has been analysed for different beam ourrent and electrodynamio structure length 
values.

2.3 The process of aooelerated partiole bunoh formation in the backward electromagnetio wave field of a corrugated waveguide has been investigated. It is found that with the onset of a steady-state regime of generation in the self-osoillation system the energy gain gain by the acoelerated partiole is of monotone oharaoter. The amount of eleotrons trapped into the the acoeleration regime makes up about $20 \%$ of the total amount of the driven REB partioles.

2.4 Estimates are given for the peak intensity of the acoelerating field and the rate of eleotron aooleration.

\section{Referenoes}

1. J.A.Nation. New Aocelerator Conoepts // Prooeedings of the 7-th Intermational Conference on High-Power Partiole Beams. 1988. V.1. P.175-183.

2. V.A.Balakirev, A.0.Ostrovsky. Charged Partiole Acoeleration by a Modulated Eleotron Beam in Corrugated Cavity // Zh.Tekh.Fiz. 1989. V.59. N6. P.142-145. (in Russ.)

3. A.0.Ostrovsky. Two-Beam Acceleration of Charged Partioles in Vaouum and Plasma Waveguide systems.// XV-th International Conferenoe on High-Energy Acoelerators. Hamburg. 1992. Abstraots. P.95

4. A.0.Ostrovsky. Dispersion and Electric Field Distribution of TM-waves in a Cylindrical Waveguide with Sinusoidal Rippled Conduction Walls // Vopr. Atom. Nauki i Tekh., ser. Tekh. Fiz. Fhksp. 1980. V.2(6). P.25-28. (in Russ.).

5. N.F.Kovalev, M.I.Petelin, M.D.Rajzer e.a. High-Power Pulse Miorowave Radiation Generated by Relativistio Electron Beam // Pis'ma Zh. Fhksp. Teor. Fiz. 1973. V.18. N.4. P.232-235. (in Russ.)

6. Y.Carmel, J.Ivers, R.E. Kribel and J.Nation.//Intense Coherent Cherenkov Radiation Due to the Interaction of a Relativistio Eleotron Beam with a Slow-Wave Struoture. // Phys.Rev.Lett. 1974. V.33. N21. P.1278-1282.

7. I.A.Vainshtein, V.A.Solntsev. Ieotures on High Frequenoy Electronios. Sov.Radio.Publ. 1973. 400p. (in Russ.)

8. V.A.Buts, V.V.Ognivenko. On a Theory of REB Cherenkor Instaability in a Wareguide with a Rippled Wall // Ukr.Fiz.Zh. 1990. V.35. N8. P.1174-1180 (in Russ.) 
9. V.I.Kurilko, V.I.Kuoherov, A.0.0strovsky, Yu.V.Tkaoh. On a Theory of REB Stability in a Cormugated Cylindrical Waveguide // Zh.Teoh.Fiz. 1979. V.49. N.12. P.2569-2575. (in Russ.).

10. N.F.Kovalev, V.I.Petrukhina, A.V.Smorgonsky. Relativistio BWO // Radioteoh. Elektron. 1975. V.20. N7. P.1547-1550. (in Russ.)

11. N.S.Ginzburg, S.P.Kuznetsor. Periodio and Stoohastio SelfModulation Regimes in Distributed Eleotron Osoillators // Relativistio RF Eleotronios. Gorky. Institute of Applied Physios. 1981. P.101-144. (in Russ.). 


\section{Figure Caption}

Fig.1 Amplitude $|A(\xi)|(a)$ and phase $\operatorname{Arg} A(\xi)$ (b) versus the longitudinal coordinate $\xi .1$ corresponds to the dependenoes obtained without the RF spaoe oharge. 2 corresponds to the dependenoes obtained with taking into aocount the RF spaoe oharge.

Fig.2 $|\tilde{A}(\xi)|(a)$ and Arg $\tilde{A}(\xi)$ (b) versus the longitudinal coordinate $\xi$.

Pig.3 Eleotron momenta $q^{a 00}$ versus the initial phases $\vartheta_{0}^{a 00}$. a) $\xi=24$, b) $\xi=48,0) \xi=72$, d) $\xi=96$.

Fig.4 Momentum of the acoelerated partiole versus the longitudinal coordinate $\xi \cdot \theta_{0}^{800}=\pi$.

Fig.5 A sketch of a periodio waveguide with acoelerating and acoelerated beams.

Fig.6 Qualitative dispersion picture of the first three radial modes of the corrugated wall wareguide.

Fig.7 Spaoe-time evolution of the backward wave amplitude $\left|A_{-}(\tau, \xi)\right|$. $v_{\mathrm{b}}=6.10^{-4}$.

Fig.8 Space distribution of the eleotrio field in a comugated waveguide. $1 .-v_{b}=6.10^{-4} ; 2 .-v_{b}=1.2 \cdot 10^{-3} ; 3 .-v_{b}=1.6 \cdot 10^{-3}$. Fig.9 Phase plane of the driven beam for $v_{b}=1.6 \cdot 10^{-3}$ and different $\xi^{\prime}$. a) $\xi^{\prime}=11$; b) $\xi^{\prime}=22$; c) $\xi^{\prime}=33$; d) $\xi^{\prime}=44$. Fig.10 Dependences of electron momenta $q^{200}$ on the initial phase $\theta_{0}^{200}$. a) $\xi^{\prime}=11$; b) $\xi^{\prime}=22$; c) $\xi^{\prime}=33$; d) $\xi^{\prime}=44$. Fig.11 Eleotron momentum $q^{a 00} \nabla s$ the longitudinal space ooordinate. Fig.12 The maximum energy gain of the acoelerated particles $\nabla s$ the driving REB ourrent $I_{0} \cdot 1 .-r_{1} / r_{0}=0.5 ; 2 .-r_{1} / r_{0}=0.3$. 


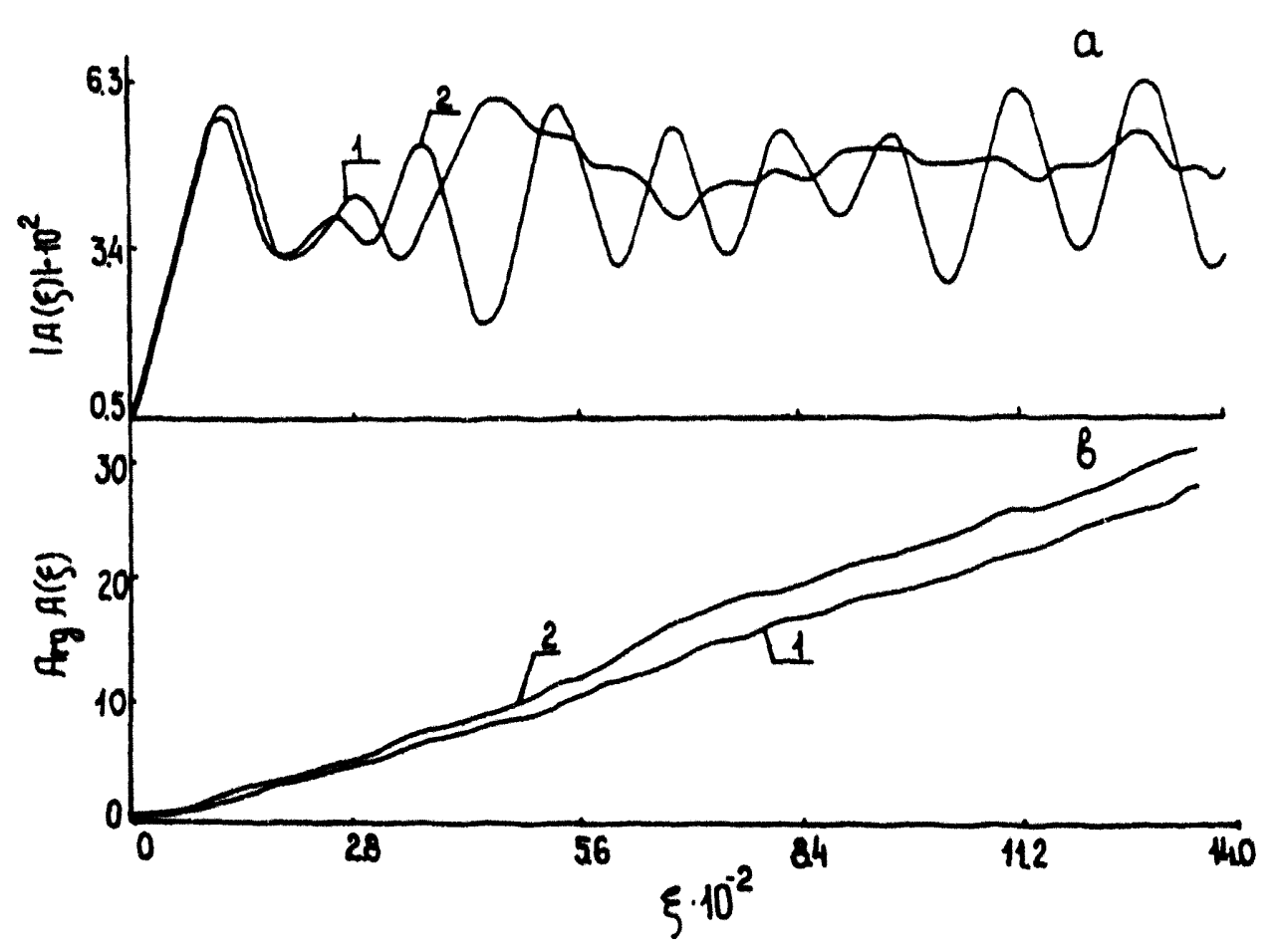

Fig. 1

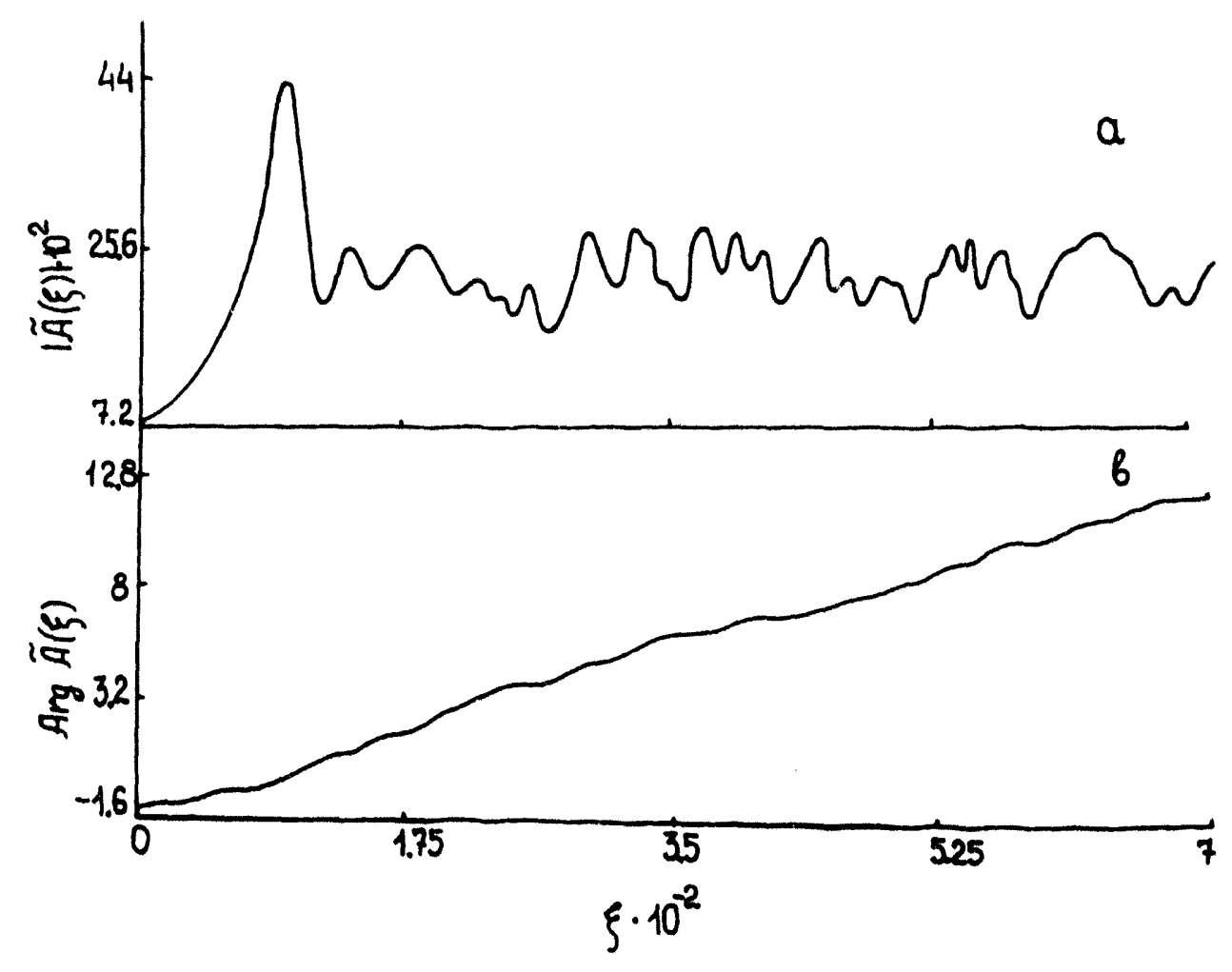

Pir. ? 


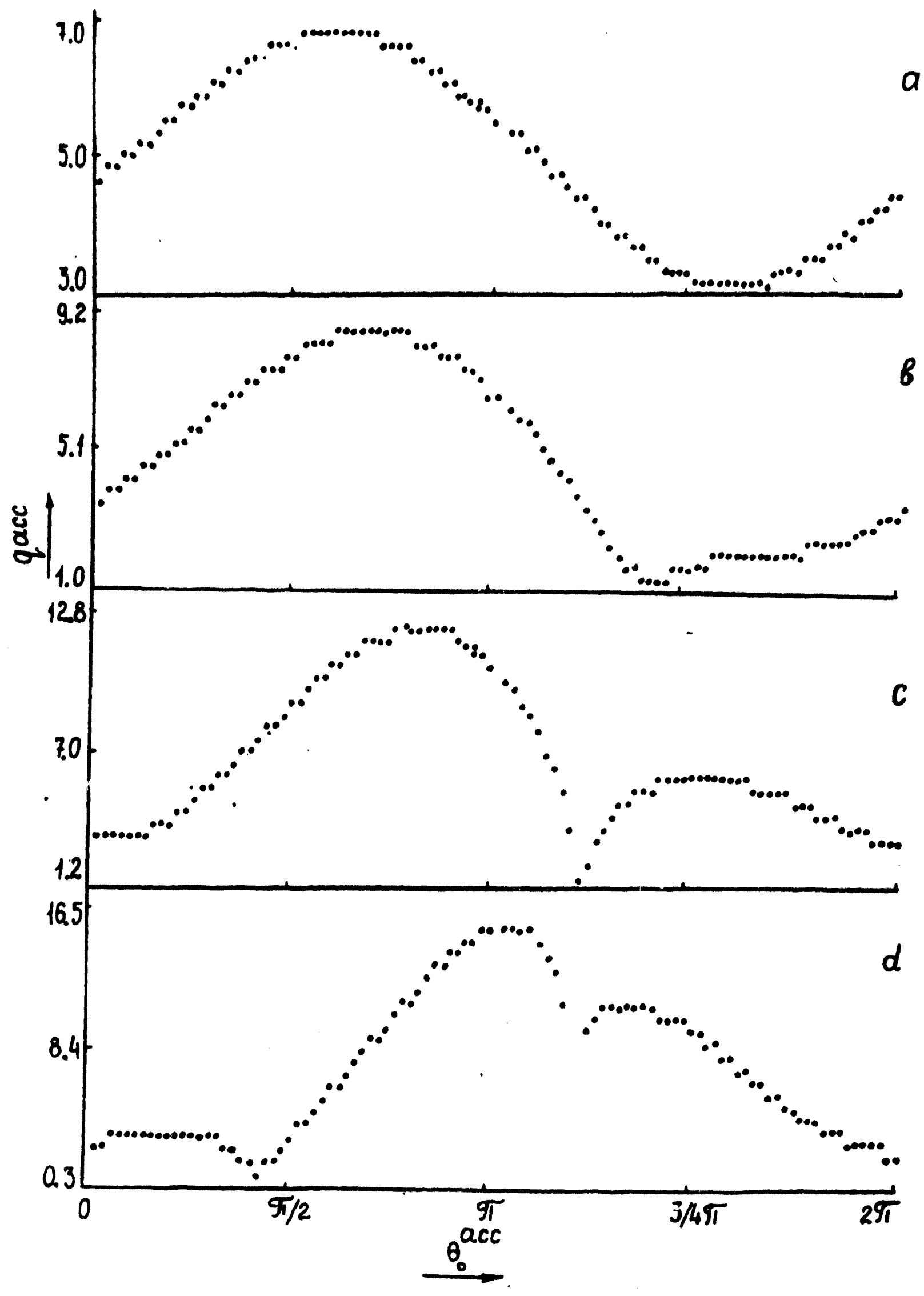

F18. 3 


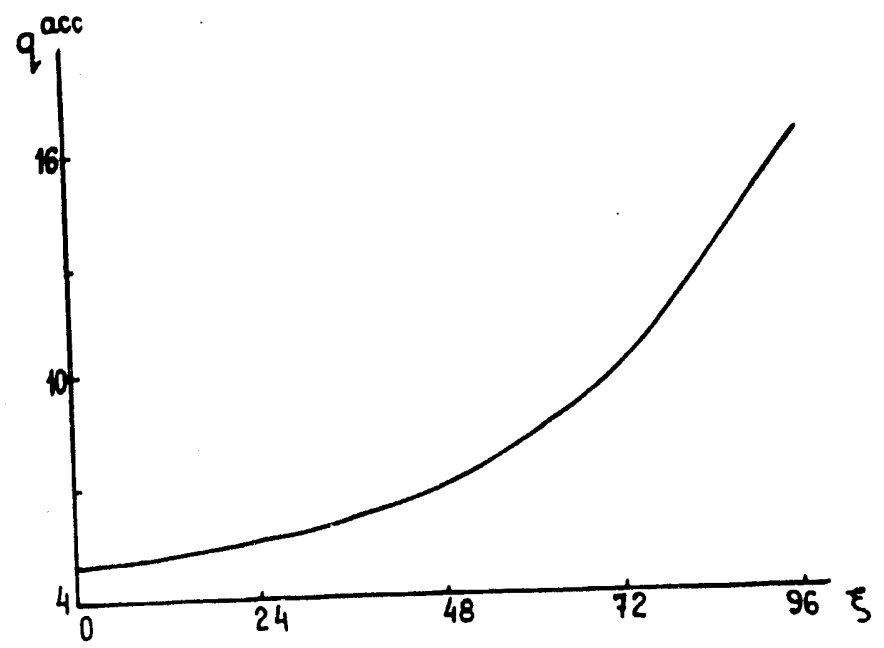

Fig. 4

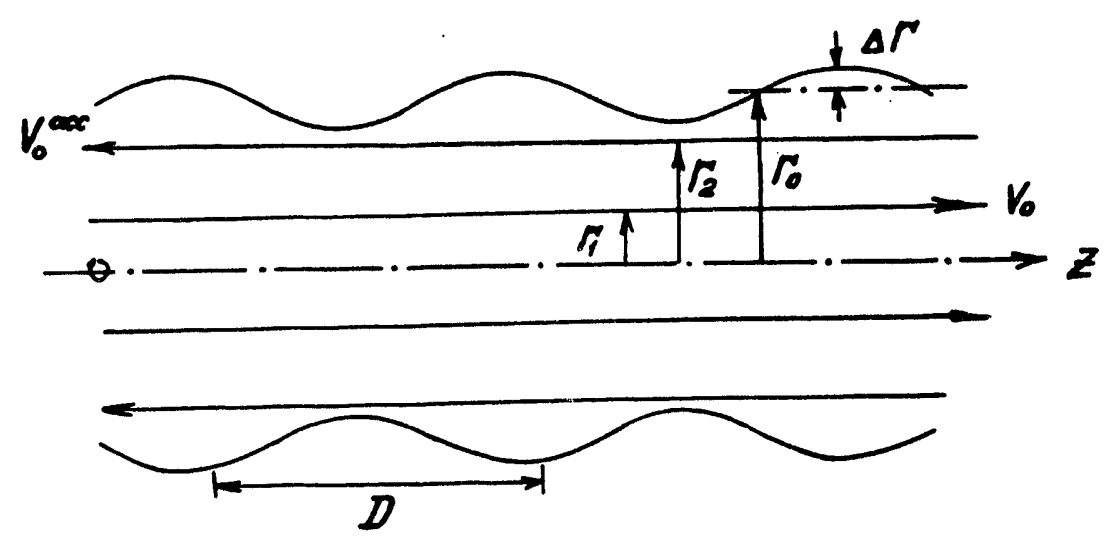

FIE. 5 


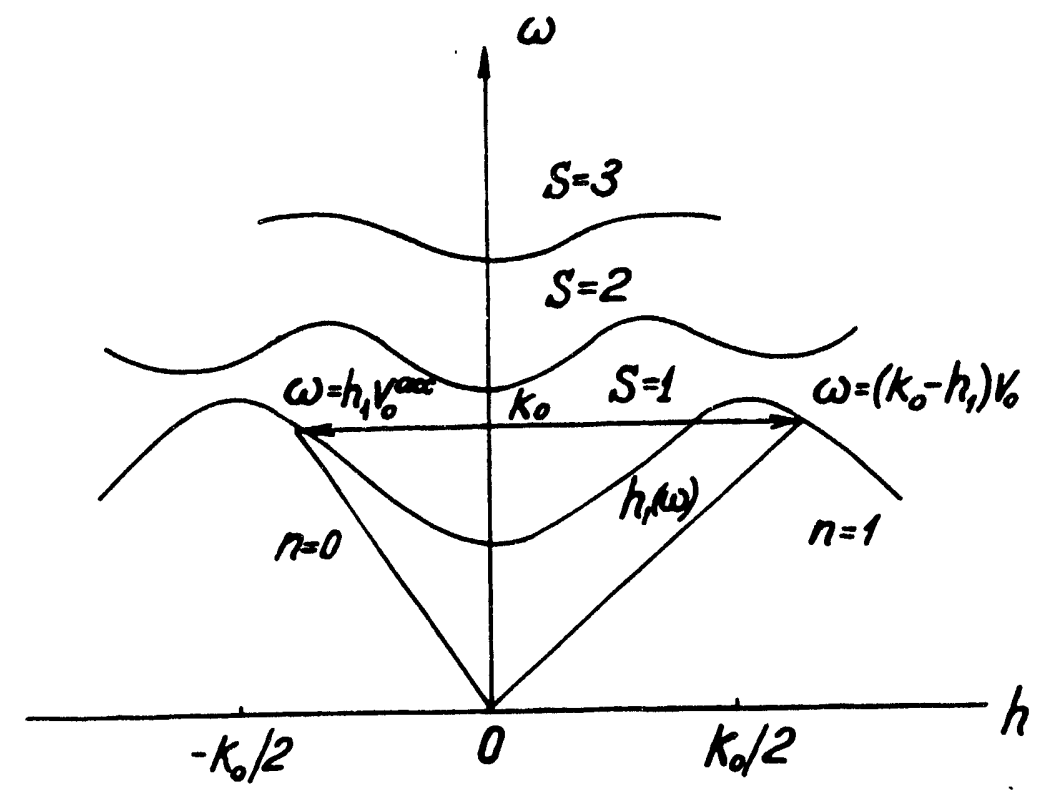

Fig. 6

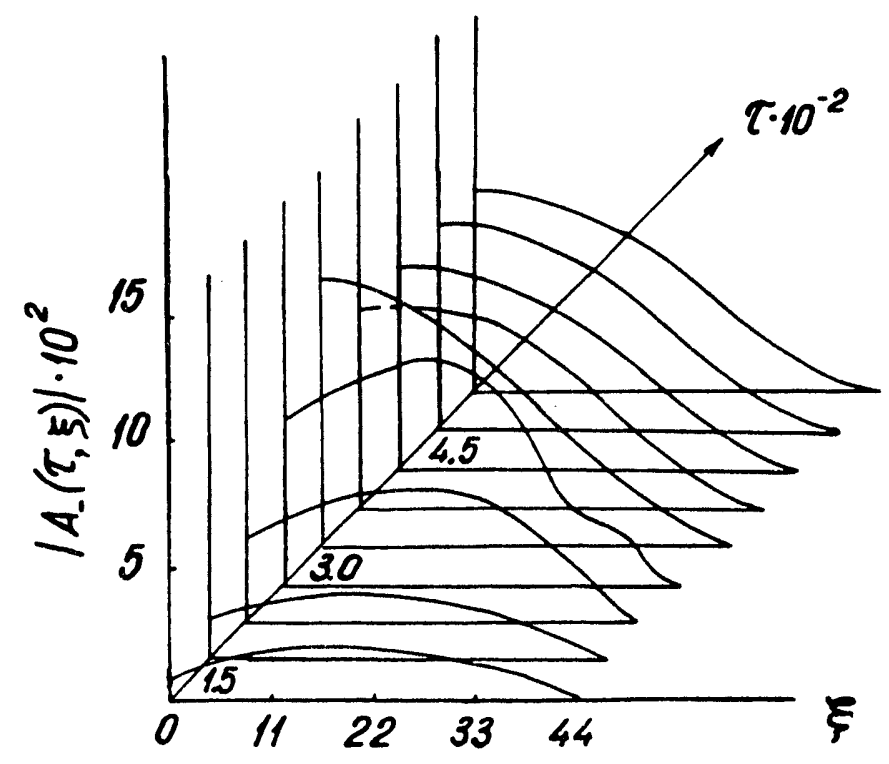

Fig. 7 


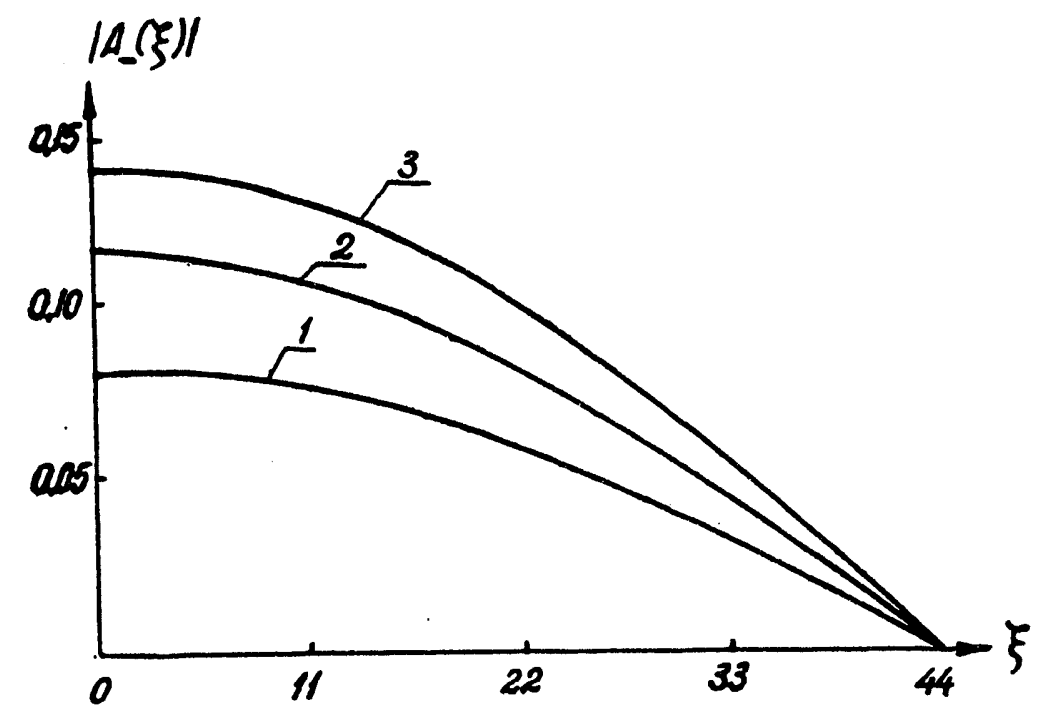

FIg. 8

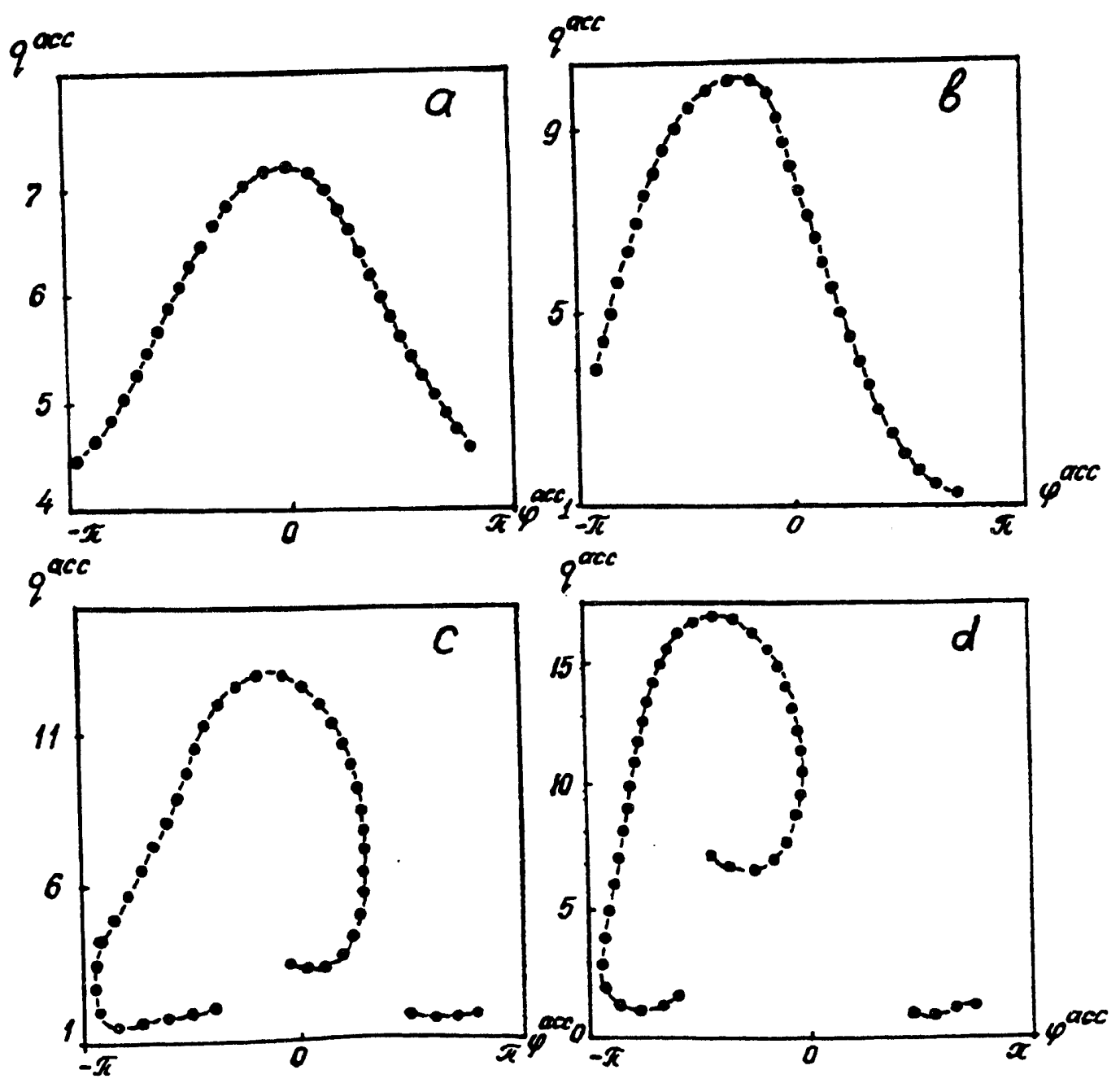

Fig. 9 


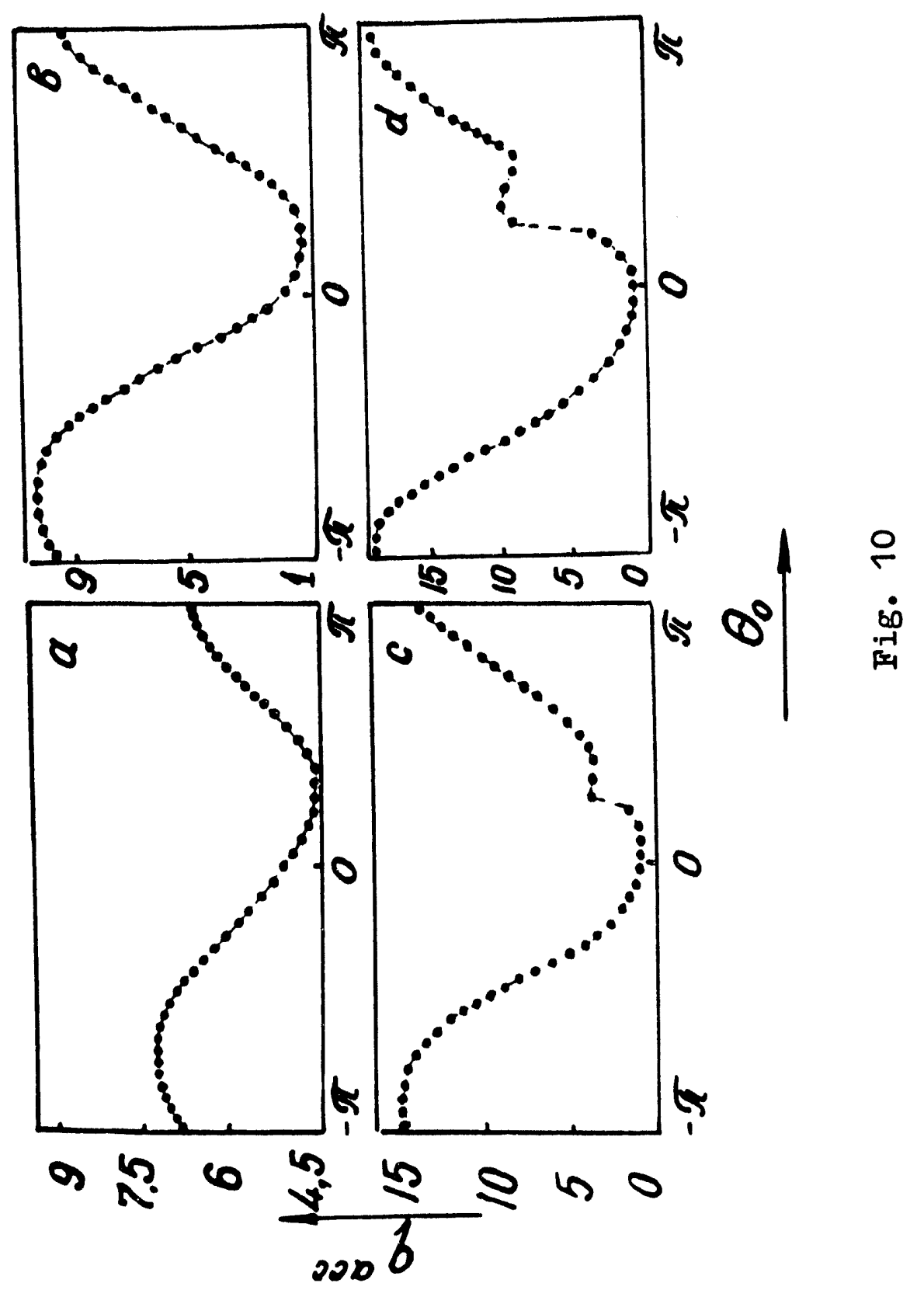




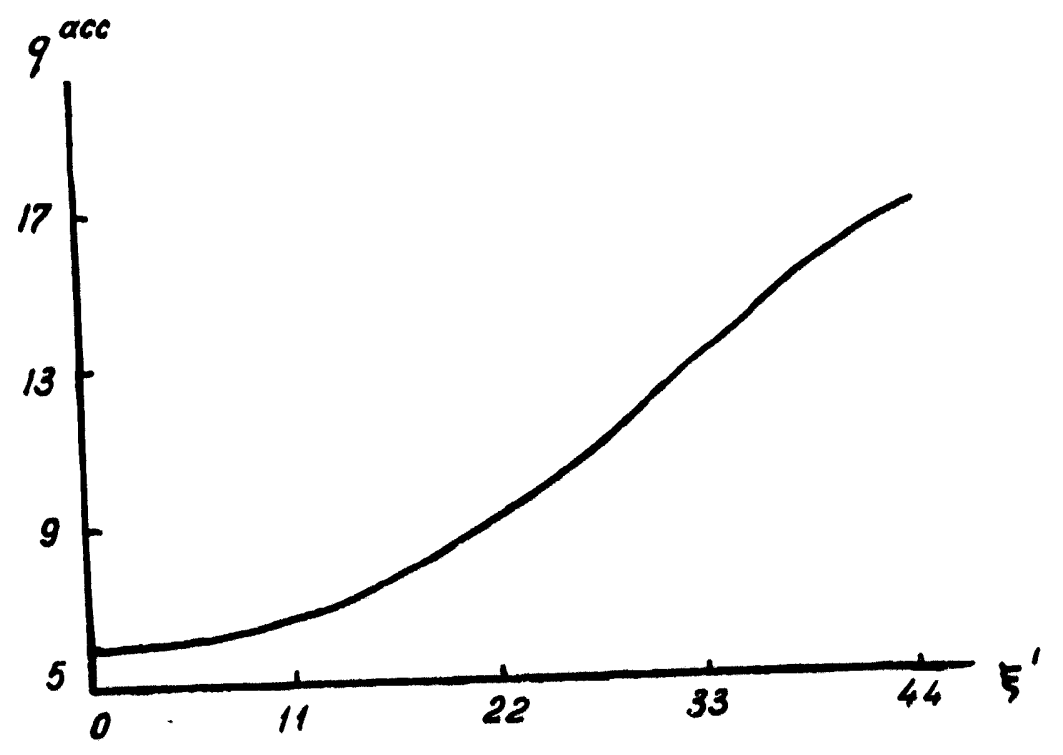

Fig. 11

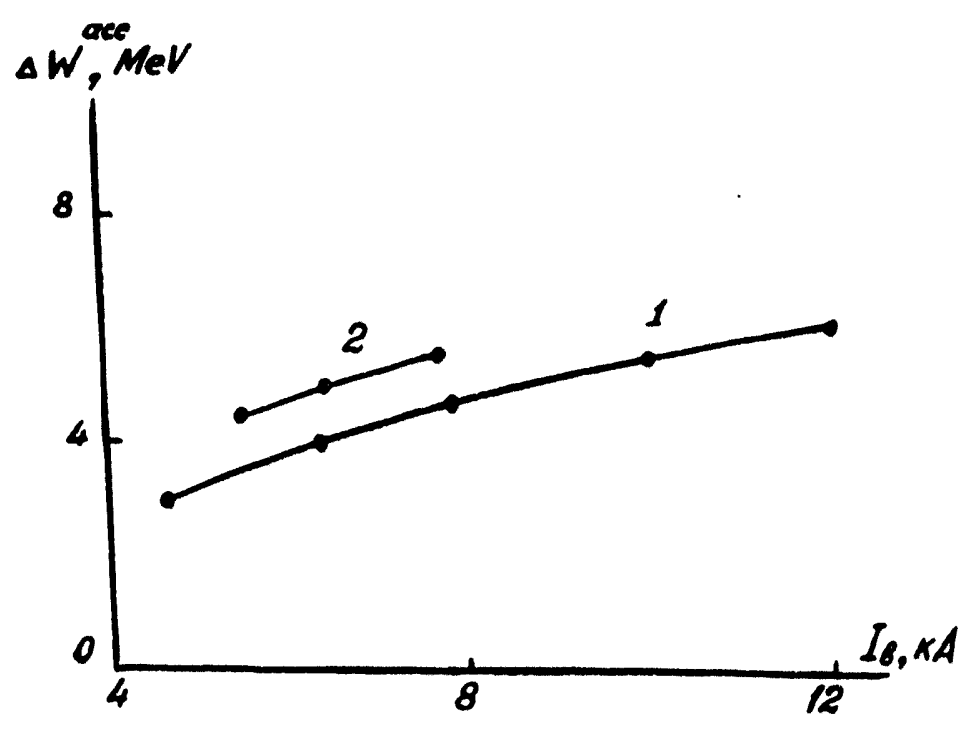

Fig. 12 

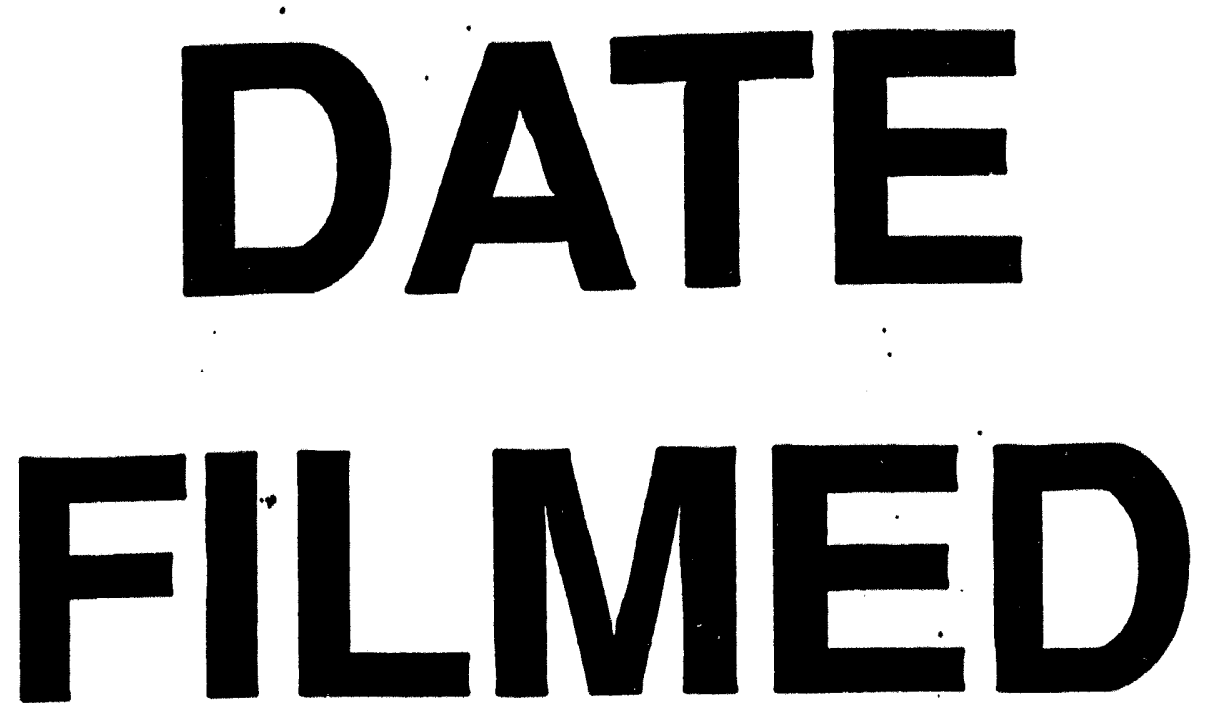

$1 / 6 / 94$
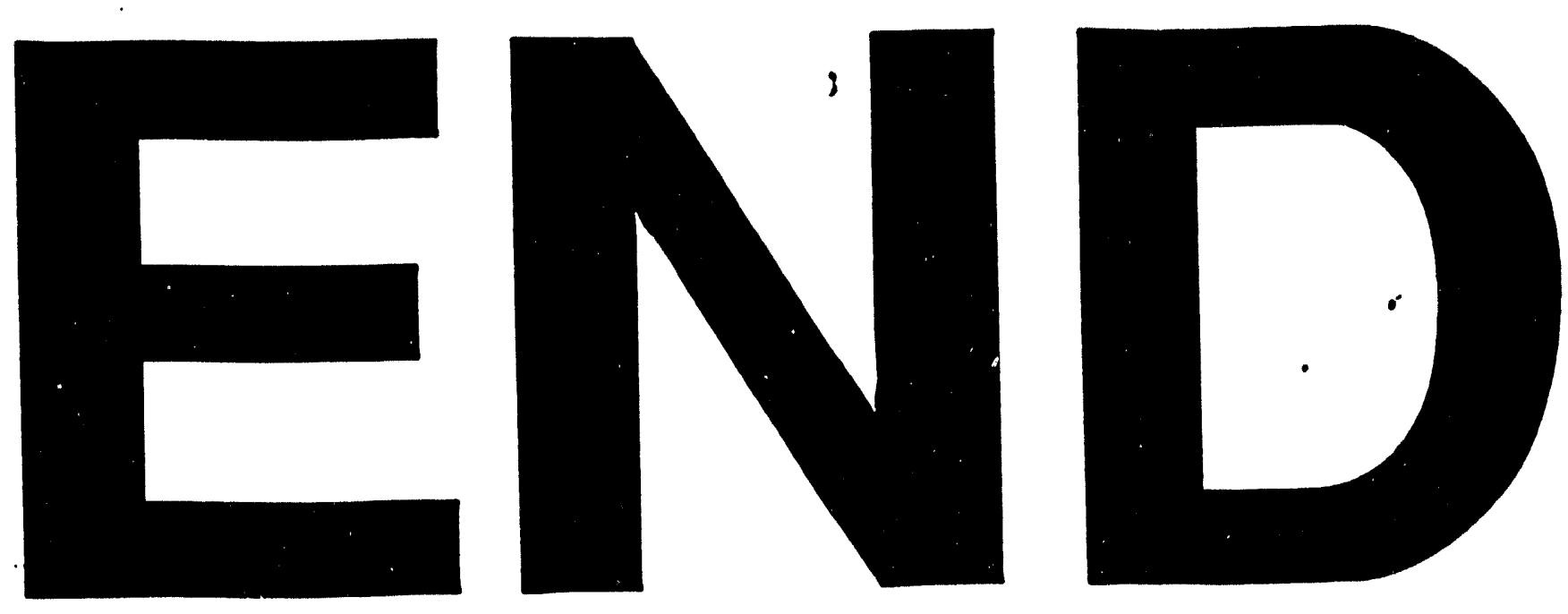
